\title{
Perfil clínico-funcional de niños que ingresan a un programa de rehabilitación oncológica en una ONG chilena
}

BEATRIZ ORMEÑO ${ }^{1}$, ANDREA VARGAS $^{2}$, MARICRUZ ORMEÑO ${ }^{3}$, CLAUDIO TORRES ${ }^{4}$.

\section{ABSTRACT \\ Clinical-functional profile of children admitted into an oncology rehabilitation program in a Chilean NGO}

Introduction: Pediatric cancer patients present several dysfunctions in their body functions and structures caused by the tumors or by therapies, such as surgery, chemotherapy, radiotherapy or the sum of all of these treatments. These dysfunctions cause impairment of all areas of functioning. Objective: To describe the clinical and functional profile of children admitted to an oncology rehabilitation program. Materials and methods: A retrospective, descriptive, observational study that reviewed 109 clinical records of patients admitted between 2014 and 2017 to the oncology rehabilitation program of 'Fundación Nuestros Hijos', in compliance with the admission criteria. Data were obtained from the clinical assessment performed by the physiatrist and from functional tests such as 'Timed Up and Go Test', 'Pediatric Balance Scale' and '6-minute walk test'. Results: Average age was $8.7 \pm 3.4$ years. The most frequent diagnoses were central nervous system (CNS) tumors (50.4\%), leukemia and lymphoma $(24.4 \%)$. A $53.2 \%$ of the patients were under active treatment at the time of admission. Motor deficit was the most frequent symptom (79.8\%). In this group, 19 children $(17.4 \%)$ had hemiparesis and 18 (16.5\%) quadraparesis. Of the total, $44 \%$ reported fatigue, $28.4 \%$ swallowing disorders, $34.9 \%$ vision disorders, and $56 \%$ cognitive impairment. Conclusions: Children admitted to oncology rehabilitation programs present several deficiencies in areas of functioning, both in early and late stages of their treatment. Motor, visual and swallowing impairment are the most common dysfunctions, particularly in patients with CNS tumors.

Key words: Dysfunction, pediatric cancer, oncology rehabilitation.

\section{RESUMEN}

Introducción: Los pacientes oncológicos infantiles presentan diversas deficiencias en sus funciones y estructuras corporales derivados de los
${ }^{1}$ Residente de Medicina Física y Rehabilitación, Facultad de Medicina CAS-UDD

${ }^{2}$ Centro de Rehabilitación Fundación Nuestros Hijos. ${ }^{3}$ Hospital Roberto del Río. ${ }^{4}$ Tecnología Médica. Universidad Andrés Bello.

Correspondencia a: Beatriz Ormeño beatrizormenou@gmail.com

Recibido: 26-3-2019 Aprobado: 15-12-2019 
tumores mismos o de los distintos tratamientos empleados, ya sea cirugía, quimioterapia, radioterapia o la suma de ellos. Estas deficiencias generan gran discapacidad en todas las áreas del funcionamiento. Objetivo: Describir el perfil clínico y funcional de niños que ingresan a un programa de rehabilitación oncológica. Materiales y Métodos: Estudio observacional, restrospectivo y descriptivo. Se revisaron 109 fichas clínicas de pacientes ingresados al programa de rehabilitación oncológica de Fundación Nuestros Hijos entre los años 2014 y 2017, que cumplieron criterios de ingreso. Se obtuvieron los datos de la evaluación clínica realizada por médico fisiatra y pruebas funcionales al ingreso, incluyendo Timed Up and Go Test, Pediatric Balance Scale y Test de Marcha de 6 min. Resultados: El promedio de edad fue de 8,7 $\pm 3,4$ años. Los diagnósticos más frecuentes fueron tumores de sistema nervioso central $(50,4 \%)$, leucemias y linfomas $(24,4 \%)$. Un $53,2 \%$ de los pacientes se encontraba en tratamiento activo al momento del ingreso. El déficit motor fue el más frecuente $(79,8 \%)$. Dentro de este grupo, 19 niños $(17,4 \%)$ presentaron hemiparesia y 18 niños $(16,5 \%)$ tetraparesia. Del total, $44 \%$ reportó presencia de fatiga, $28,4 \%$ presentó trastorno de la deglución, $34,9 \%$ trastorno visual y un $56,9 \%$ compromiso cognitivo. Conclusiones: Los niños que ingresan a rehabilitación oncológica presentan múltiples deficiencias en diversas áreas de su funcionamiento, tanto en etapas tempranas como tardías de su tratamiento. El compromiso, motor, deglutorio, visual y cognitivo son las deficiencias más frecuentes de encontrar, sobre todo en pacientes con tumores de SNC.

Palabras clave: Deficiencia, cáncer infantil, rehabilitación oncológica.

\section{Introducción}

El estudio del Observatorio Global contra el Cáncer (GLOBOCAN) del año 2012 demostró un aumento en la incidencia de cáncer infantil en países en vías de desarrollo $(147,000$ cánceres/año) ${ }^{1}$.

En nuestro país se presentan aproximadamente 500 nuevos casos de cáncer infantil por año con una sobrevida global de $71,4 \%$ a los cinco años de realizado el diagnóstico. Las neoplasias más comunes en los niños son las hematológicas y linfáticas (leucemias y linfomas) sumando un 50\%, seguidas de los tumores de sistema nervioso central (SNC) que corresponden al 15,9\%. Menos frecuentes, son los tumores de partes blandas y tumores óseos ${ }^{2}$.

La clasificación internacional del funcionamiento y la discapacidad en su versión para niños y adolescentes (CIF-CY) es el lenguaje universalmente propuesto para documentar el funcionamiento en sobrevivientes de cán- cer y como marco de trabajo en un plan de rehabilitación ${ }^{3}$. En esta línea de trabajo, se han logrado establecer core sets específicos, con las principales alteraciones en el funcionamiento, para pacientes con tumores de sistema nervioso central ${ }^{4}$. Las deficiencias de funciones y estructuras corporales observadas en oncología pueden derivar del cáncer mismo o de las diferentes modalidades terapéuticas como cirugía, radioterapia y quimioterapia. Los tratamientos oncológicos abarcan largos períodos y es posible evidenciar deficiencias durante el transcurso de éstos. Las terapias de rehabilitación en este tipo de pacientes han demostrado beneficios tanto en el ámbito funcional como de calidad de vida.

Las secuelas a largo plazo en sobrevivientes de cáncer infantil se encuentran bien descritas y son principalmente alteraciones endocrinas, musculoesqueléticas, neurológicas y cardiopulmonares, entre otras ${ }^{4}$. Equilibrio, capacidad cardiovascular, percepción de fatiga 
y densidad ósea, entre otros, son variables que se ha demostrado que mejoran en programas de rehabilitación ${ }^{5,6}$. Sin embargo, no están descritas las deficiencias que presentan durante sus tratamientos o en etapas tempranas post tratamiento.

En Chile, el año 2014, se inaugura el Centro de Rehabilitación Oncológico de la Fundación Nuestros Hijos (CROFNH). Esta fundación es una ONG chilena dirigida a apoyar niños con patología oncológica y a sus familias. El equipo rehabilitador lo integran kinesiólogos, terapeutas ocupacionales, fonoaudiólogos, neuropsicólogos, odontólogo, educador diferencial, nutricionista, trabajador social y médico fisiatra. Los pacientes atendidos provienen de unidades oncológicas que son centros de referencia hospitalarios para todo Chile y derivan pacientes precozmente. Al ingreso a CROFNH, según un protocolo establecido, los niños son evaluados integralmente y se establece un diagnóstico funcional y los objetivos de rehabilitación.

La rehabilitación oncológica es un área emergente dentro de la fisiatría en el mundo ${ }^{7}$. Esta disciplina ha podido definir perfiles clínicos de pacientes según ciertas características como el tipo de cáncer y los tratamientos recibidos. Uno de los perfiles más estudiados en el adulto, es el de mujeres sobrevivientes a cáncer de mama, donde ya se conocen las probables deficiencias a encontrar y, por ende, las intervenciones más frecuentes de realizar8.

El objetivo del presente estudio es describir el perfil clínico y principales deficiencias encontradas en niños que ingresan a un programa de rehabilitación oncológica.

\section{Materiales y Métodos}

Estudio en base a serie de casos, descriptivo y retrospectivo. Fueron incluidos niños y adolescentes de hasta 18 años, con diagnóstico oncológico, que ingresaron a CROFNH entre septiembre de 2014 y agosto de 2017 y que contaban con evaluación de ingreso completa en su ficha clínica. Se excluyeron pacientes con evaluación de ingreso incompletas, fallecidos previos al reclutamiento y aquellos en que no se pudo obtener el consentimiento informado.

Este estudio fue aprobado por el Comité Ético Científico de la Facultad de Medicina Clínica Alemana de Santiago-Universidad del Desarrollo (CAS-UDD) y por el Comité de Investigación y Desarrollo de la Fundación Nuestros Hijos. Se solicitó consentimiento informado vía telefónica a los tutores de pacientes y asentimiento a niños entre $12 \mathrm{y}$ 18 años.

Se revisaron las fichas clínicas electrónicas de pacientes y se recopiló información demográfica (edad, género, previsión de salud) e información clínica del ingreso (diagnóstico, estado nutricional, tratamientos recibidos, evaluaciones realizadas por otros especialistas, necesidad de ayudas técnicas). Se registraron las deficiencias adquiridas hasta ese momento en base a la evaluación clínica por médico fisiatra. En algunos casos, frente a la sospecha clínica, se estudió el trastorno cognitivo con la Escala de Weschler y la polineuropatía con estudio electrodiagnóstico.

También se registró el protocolo de evaluación de ingreso que comprende las siguientes evaluaciones funcionales, todas las cuales se expresan en porcentaje del teórico esperado para la edad:

a) Timed Up and Go Test (TUG) $)^{9}$ : evalúa movilidad funcional y transferencias y se puede realizar desde los 3 años de edad. Se mide el tiempo que el paciente demora en levantarse de una silla, caminar una distancia estandarizada y vuelve a sentarse. Posee valores normalizados para las distintas edades.

b) Test de marcha de 6 minutos $(M 6 M)^{10}$ : aplicable desde los 6 años de edad, pues requiere comprensión de instrucciones. Ampliamente usada como medición de resistencia cardiovascular en adultos y en niños con cáncer y se ha validado su correlación con la capacidad aeróbica del individuo ( $\mathrm{VO}_{2}$ máxima). Posee valores estandarizados por edad.

c) Escala de Equilibrio Pediátrico $(P B S)^{11}$ : puede aplicarse desde los 4 años de edad, posee valores estandarizados por edad. Los puntajes mínimo y máximo son 0 y 56 . Las pruebas funcionales se registran en porcentaje respecto a lo esperado por edad. 
Se registró, además, la presencia de fatiga (sí/no) y la derivación a los distintos profesionales del equipo de rehabilitación indicada al ingreso a FNH por fisiatra.

Se clasificó a los pacientes de acuerdo a la etapa de tratamiento en que se encontraban al ingreso, ya sea tratamiento activo (quimioterapia activa o intensiva en curso), etapa de mantención (quimioterapia de mantención), seguimiento (etapa sin intervenciones terapéuticas, solo en control hasta generalmente 5 años postratamientos), en cuidados paliativos (CCPP) o de alta de seguimiento por oncología. Se registraron complicaciones adquiridas durante el proceso de enfermedad, derivadas del tumor mismo o de tratamientos recibidos, tales como trastornos endocrinos, dolor, polineuropatía periférica (PNP) de distintos orígenes y necrosis avascular de huesos largos. Se elaboró una planilla Excel donde se registraron los

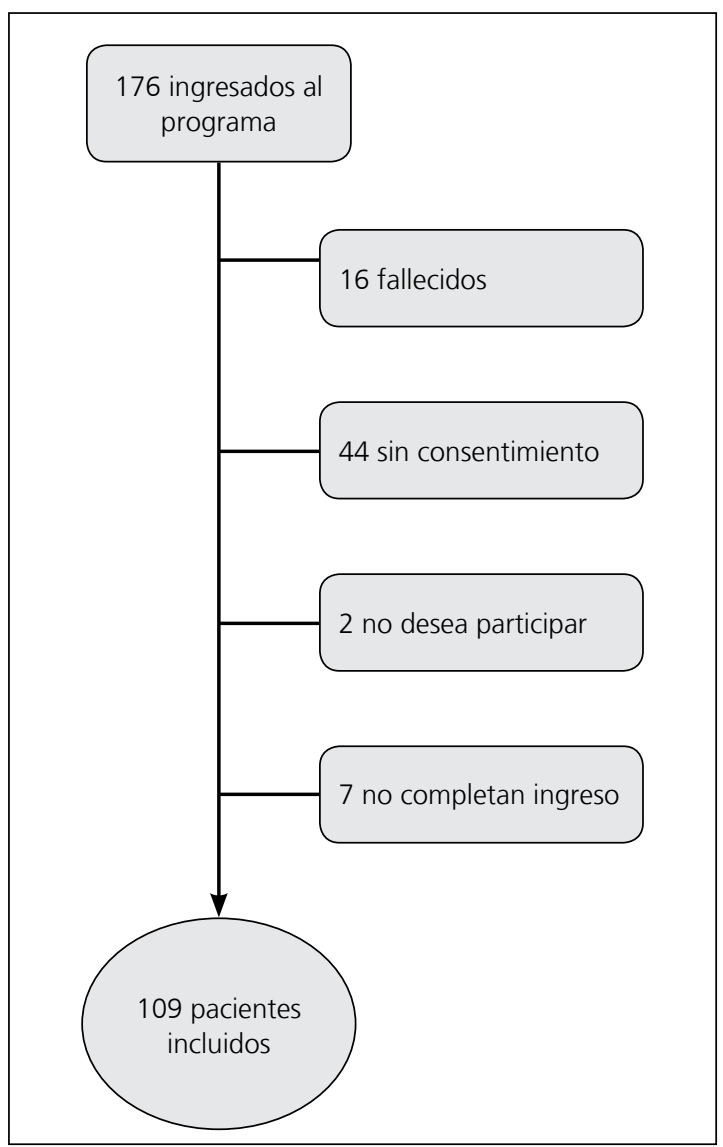

Figura 1. Pacientes incluidos y excluidos del estudio. datos y se calcularon estadísticas descriptivas de posición y dispersión.

\section{Resultados}

De un total de 176 ingresados en este período, se incluyeron 109 pacientes $(61,9 \%)$ en el estudio. De los 67 excluidos, 16 fallecieron, 7 no completaron el ingreso formal, 44 no se lograron contactar para consentimiento y dos pacientes se negaron a participar del estudio. Las características del grupo estudiado se describen en Tabla 1. Las edades al momento del ingreso variaron entre tres meses a dieciocho años con un promedio de $8,7 \pm 3,4$ años. Un 53\% correspondía a pacientes de sexo masculino. La mayoría de los pacientes $(43,1 \%)$ pertenecían a FONASA del tramo A. $87 \%$ residía en la Región Metropolitana y la mayoría se atendía en centros de referencia de los Servicios de Salud Metropolitanos Norte y Sur.

El diagnóstico oncológico más frecuente fue tumor de SNC $(50,4 \%)$ y la mayoría correspondía a tumores de fosa posterior; seguido de neoplasias hematológicas $(24,4 \%)$, principalmente leucemias y linfomas. 53,2\% de los pacientes se encontraba en fase de tratamiento activo al momento del ingreso. $33 \%$ presentó desnutrición a su ingreso y un $16,5 \%$ sobrepeso u obesidad. $45 \%$ recibió un tratamiento, ya sea quimioterapia, radioterapia o cirugía. El resto de los pacientes recibió dos o tres tratamientos resultado de la combinación de los anteriores. Sólo el 4,6\% no recibió ningún tratamiento.

\section{Deficiencias}

Las deficiencias registradas al ingreso a rehabilitación se muestran en Tabla 2, encontrándose múltiples deficiencias por paciente.

En el grupo de cánceres hematológicos primó la fatiga $(77,4 \%)$ seguido del compromiso motor $(64,5 \%)$ y en el grupo de tumores de SNC, destacó el compromiso motor $(92,7 \%)$ y el cognitivo $(65,5 \%)$.

La deficiencia más frecuente entre todos los diagnósticos fue la motora con un 79,8\%. Un 56,9\% presentó deficiencia cognitiva, incluyendo bajo funcionamiento intelectual, déficit de memoria, déficit de atención o baja velocidad de procesamiento. La intolerancia 
Tabla 1. Características demográficas y clínicas de la población estudiada

\begin{tabular}{|c|c|c|}
\hline Variable & $\mathbf{n}$ & $\%$ \\
\hline Total & 109 & 100,0 \\
\hline \multicolumn{3}{|l|}{ Sexo } \\
\hline Femenino & 51 & 46,7 \\
\hline Masculino & 58 & 53,2 \\
\hline \multicolumn{3}{|l|}{ Edad al ingreso (años) } \\
\hline $0-4$ & 25 & 22,9 \\
\hline $5-9$ & 39 & 35,7 \\
\hline $10-18$ & 45 & 41,2 \\
\hline Edad (promedio \pm DS) & \multicolumn{2}{|c|}{$8,7 \pm 3,4$} \\
\hline \multicolumn{3}{|l|}{ Previsión } \\
\hline Fonasa A & 47 & 43,1 \\
\hline Fonasa B & 29 & 26,6 \\
\hline Fonasa C & 19 & 17,4 \\
\hline Fonasa D & 12 & 11,0 \\
\hline Isapre & 2 & 1,8 \\
\hline \multicolumn{3}{|l|}{ Diagnóstico } \\
\hline Tumores de SNC & 55 & 50,4 \\
\hline Leucemia-linfoma & 31 & 24,4 \\
\hline Sarcomas & 9 & 8,2 \\
\hline Otros & 14 & 12,8 \\
\hline \multicolumn{3}{|c|}{$\mathbf{N}^{\circ}$ de tratamientos recibidos* } \\
\hline 0 & 5 & 4,6 \\
\hline 1 & 49 & 45,0 \\
\hline 2 & 26 & 23,9 \\
\hline 3 & 29 & 26,6 \\
\hline \multicolumn{3}{|c|}{ Etapa de tratamiento al ingreso } \\
\hline Activo & 58 & 53,2 \\
\hline Seguimiento & 21 & 19,2 \\
\hline Mantención & 27 & 24,7 \\
\hline Cuidados paliativos & 2 & 1,8 \\
\hline Alta & 1 & 0,9 \\
\hline \multicolumn{3}{|c|}{ Estado nutricional al ingreso } \\
\hline Desnutrición & 36 & 33,0 \\
\hline Normopeso & 37 & 33,9 \\
\hline Sobrepeso u obesidad & 18 & 16,5 \\
\hline No registrado & 18 & 16,5 \\
\hline
\end{tabular}

*Suma entre quimioterapia, radioterapia y/o cirugía. al ejercicio o fatigabilidad estuvo presente en $44,0 \%$ del total.

En cuanto a deficiencias sensoriales, $34,9 \%$ presentó trastorno visual, como estrabismo, compromiso de par craneano visual o déficit de campo visual. $22,9 \%$ presentó hipoacusia. $27,5 \%$ reportó presencia de dolor y un $26,6 \%$ de los niños presentó algún tipo de trastorno hormonal.

Los distintos tipos de deficiencias motoras encontradas se muestran en Tabla 3. La deficiencia motora más frecuente fue hemiparesia $(17,4 \%)$ seguido de tetraparesia $(16,5 \%)$. Un $11,9 \%$ presentó compromiso motor secundario a polineuropatía por drogas o del paciente crítico. Ocho niños $(7,3 \%)$ presentaron retraso de la adquisición de hitos motores. Tres pacientes presentaron disfunción de la articulación temporomandibular secundaria a necrosis avascular del cóndilo mandibular.

$18 \%$ de los pacientes ingresados eran portadores de germen multirresistente y se atendían con medidas de aislamiento. 39\% requería de ayudas técnicas al momento del ingreso, siendo la silla de ruedas la más requerida.

\section{Pruebas funcionales}

En la Tabla 4 se muestran los resultados obtenidos en las pruebas aplicadas. El rendimiento se expresa en porcentaje de lo esperado por edad, fluctuando el promedio entre el $69,0 \%$ y el $87,0 \%$. Las pruebas no fueron aplicables a cerca de la mitad de los niños debido a limitaciones en la movilidad, por no cumplimiento de los requisitos de edad o por falta de cooperación debido a trastornos conductuales o cognitivos. No se encontraron diferencias de rendimiento en las pruebas funcionales según el tipo de cáncer.

\section{Terapias de rehabilitación indicadas}

Las terapias más indicadas fueron kinesioterapia y terapia ocupacional en $85 \%$ y $75 \%$ de las evaluaciones iniciales. Fonoaudiología se indicó en $50 \%$ de los ingresos y neuropsicología en $60 \%$.

Dos pacientes fueron derivados al momento del diagnóstico y en ellos se realizó evaluaciones iniciales y rehabilitación antes 
Tabla 2. Deficiencias encontradas al ingreso según diagnóstico

\begin{tabular}{|c|c|c|c|c|c|c|c|c|c|c|}
\hline \multirow[b]{2}{*}{ Tipo de deficiencia } & \multicolumn{10}{|c|}{ Número de deficiencias según diagnóstico } \\
\hline & Total & $\%$ & $\begin{array}{c}\text { Tumores } \\
\text { SNC }\end{array}$ & $\%$ & $\begin{array}{l}\text { Hemato- } \\
\text { lógicas }\end{array}$ & $\%$ & Sarcomas & $\%$ & Otros & $\%$ \\
\hline Total niños & 109 & 100,0 & 55 & 100,0 & 31 & 100,0 & 9 & 100,0 & 14 & 100,0 \\
\hline $\begin{array}{l}\text { Motora (origen central y/o } \\
\text { periférico) }\end{array}$ & 87 & 79,8 & 51 & 92,7 & 20 & 64,5 & 6 & 66,7 & 10 & 71,4 \\
\hline Cognitiva & 62 & 56,9 & 36 & 65,5 & 15 & 48,4 & 2 & 22,2 & 9 & 64,3 \\
\hline Fatigabilidad & 48 & 44,0 & 23 & 41,8 & 24 & 77,4 & - & - & 1 & 7,1 \\
\hline Endocrino & 29 & 26,6 & 22 & 40,0 & 4 & 12,9 & 1 & 11,1 & 3 & 21,4 \\
\hline Del habla & 48 & 44,0 & 31 & 56,4 & 11 & 35,5 & 2 & 22,2 & 4 & 28,6 \\
\hline Deglutoria & 31 & 28,4 & 18 & 32,7 & 6 & 19,4 & 2 & 22,2 & 5 & 35,7 \\
\hline Visual & 38 & 34,9 & 31 & 56,4 & 3 & 9,7 & 1 & 11,1 & 3 & 21,4 \\
\hline Dolor & 30 & 27,5 & 10 & 18,2 & 10 & 32,3 & 4 & 44,4 & 6 & 42,9 \\
\hline Del lenguaje & 34 & 31,2 & 18 & 32,7 & 9 & 29,0 & - & - & 7 & 50,0 \\
\hline Auditiva & 25 & 22,9 & 22 & 40,0 & 1 & 3,2 & - & - & 2 & 14,3 \\
\hline Total deficiencias & 432 & 100,0 & 262 & 54,4 & 103 & 21,4 & 18 & 3,7 & 50 & 10,4 \\
\hline$N^{\circ}$ deficiencias/niño & 4,0 & & 4,8 & & 3,3 & & 2,0 & & 3,6 & \\
\hline
\end{tabular}

Tabla 3. Tipo de trastorno motor en población estudiada

\begin{tabular}{|lrr|}
\hline Tipo trastorno motor & $\mathbf{n}$ & \% \\
\hline Ninguno & 22 & 20,2 \\
\hline Hemiparesia & 19 & 17,4 \\
\hline Tetraparesia & 18 & 16,5 \\
\hline Secundario asociado a PNMP & 13 & 11,9 \\
\hline Síndrome cerebeloso & 12 & 11,0 \\
\hline Paraparesia & 10 & 9,2 \\
\hline RDSM & 8 & 7,3 \\
Monoparesia & 4 & 3,7 \\
\hline Disfunción ATM & 3 & 2,8 \\
\hline Total & 109 & 100,0 \\
\hline
\end{tabular}

${ }^{\star} \mathrm{PNMP}=$ polineuromiopatía. ${ }^{*} \mathrm{RDSM}=$ retraso desarrollo psicomotor. ${ }^{*}$ ATM $=$ articulación temporomandibular. de iniciar algún tipo de tratamiento oncológico (prehabilitación).

\section{Discusión}

\section{Generalidades de la población estudiada}

Este es el primer estudio que describe las deficiencias que se presentan durante y posterior a los tratamientos oncológicos en una población de niños chilenos, ingresados a un programa de rehabilitación.

En el grupo estudiado el diagnóstico más frecuente fue tumores de SNC y en segundo lugar tumores hematológicos siendo que la prevalencia de éstos es inversa. Esto pudiese

Tabla 4. Resultado de pruebas funcionales expresado en porcentaje del teórico esperado para la edad

\begin{tabular}{|lccccccc|}
\hline Prueba & n & $\begin{array}{c}\text { Pacientes evaluados } \\
\% *\end{array}$ & $\begin{array}{c}\text { Valor prueba } \\
\text { media } \pm \text { DS }\end{array}$ & n & Pacientes no evaluados \\
Por edad & $\begin{array}{c}\text { Por razones } \\
\text { clínicas }\end{array}$ \\
\hline TUG (\%) & 64 & 58,7 & $76,0 \pm 25$ & 45 & 41,3 & $12(<3$ años) & 33 \\
PBS (\%) & 55 & 50,5 & $87,0 \pm 15$ & 54 & 49,5 & $20(<4$ años) & 34 \\
M6M (\%) & 45 & 41,3 & $69,0 \pm 24$ & 64 & 58,7 & $35(<6$ años) & 29 \\
\hline
\end{tabular}

*Porcentaje calculado en base al total de pacientes del estudio $=109$. 
explicarse por la mayor necesidad de rehabilitación de los pacientes con tumores de SNC, lo que está descrito en la literatura ${ }^{12}$. Por otro lado, aquellos pacientes con deficiencias menores, que no requirieron rehabilitación, podrían verse sobre presentados en este estudio debido a que no fueron derivados desde sus respectivos centros oncológicos, o bien, no se detectaron sus deficiencias por el equipo tratante.

La edad se encontró uniformemente distribuida entre rangos etarios. Estudios de incidencia muestran que el grupo más afectado es el de 0 a 4 años ${ }^{2}$, por lo que suponemos que un grupo de niños pequeños no es derivado ya sea porque no presenta deficiencias o en ellos pudiese subestimarse la necesidad de rehabilitación.

La proporción de hombres es levemente mayor al de mujeres, al igual como se ha encontrado en estudios de poblacionales de cáncer en $\mathrm{Chile}^{2}$. Se desconoce los motivos de esta diferencia.

La mayoría de los pacientes del estudio ingresó tempranamente a rehabilitación, en etapa de tratamiento activo. Esto hace particularmente interesante el estudio, ya que podemos registrar las deficiencias que presenta esta población a corto plazo y que no son descritas en la literatura. Cuando observamos las secuelas que se describen a largo plazo en estos pacientes, muchas ya estaban presentes en nuestro grupo al ingreso (trastornos motores, sensoriales, cognitivos). Esto nos reafirma la necesidad temprana de rehabilitación en esta patología.

La literatura, actualmente, respalda la prehabilitación, ya que se han observado mejores outcomes cuando se inician programas muy tempranamente ${ }^{13}$.

Es importante recalcar que la malnutrición, ya sea por déficit o por exceso, afectó a $2 / 3$ del grupo estudiado, esto se ha observado en otros grupos de estudio ${ }^{14}$. Estas cifras recalcan la importancia de prevenir esta comorbilidad y de contar con nutricionista en el equipo rehabilitador.

\section{Trastornos musculoesqueléticos}

La deficiencia motora fue la más frecuente en el grupo estudiado. La etiología de la deficiencia neuromotora en los pacientes oncoló- gicos es multifactorial. Puede producirse por daño directo de áreas cerebrales involucradas en la ejecución del movimiento, de nervios periféricos, producto de desacondicionamiento general o por la suma de estas alteraciones ${ }^{15}$. El trastorno de equilibrio y coordinación, con mayor compromiso de extremidades inferiores ha sido descrito en los pacientes con cáncer. En pacientes con tumores de SNC, esto se atribuye principalmente a un síndrome cerebeloso. No obstante, existen otros factores que pueden originar este déficit, como polineuropatía de diversas etiologías (por corticoides, del paciente crítico), miopatía por corticoides y desacondicionamiento muscular.

El porcentaje de osteonecrosis sintomática descrito en la literatura se encuentra entre 1\% y $9 \%$ de todos los niños tratados por leucemia, lo que concuerda con nuestros resultados ${ }^{4}$.

Mientras no se desarrollen tratamientos que generen menores secuelas motoras, consideramos crucial que los pacientes reciban rehabilitación temprana como parte fundamental de sus tratamientos. La rehabilitación debiese iniciarse al momento del diagnóstico, de manera de alcanzar a realizar prehabilitación, e idealmente, con un equipo con experiencia en manejo de pacientes oncológicos.

\section{Trastorno cognitivo}

En grupos de sobrevivientes de cáncer infantil se ha encontrado que un tercio o más de ellos presentan algún tipo de trastorno cognitivo. En nuestro estudio, un 56,9\% presentó trastorno cognitivo, lo que concuerda con otros grupos ${ }^{16}$. El trastorno cognitivo fue diagnosticado en la evaluación clínica inicial y confirmado por psicometría en algunos casos, lo que podría haber generado un sesgo de entrevistador a la hora de estimar la prevalencia de este trastorno.

El trastorno cognitivo en estos pacientes puede ser transitorio o permanente y se presume que su origen sería multifactorial. Se sabe que el volumen de radiación recibida y la edad en que el paciente fue tratado son los principales factores que determinan secuelas neuropsicológicas ${ }^{15-17}$. El término "Chemobrain" se ha acuñado para la disfunción cognitiva transitoria posterior a la 
quimioterapia donde principalmente se ven afectadas memoria, concentración y velocidad de procesamiento ${ }^{20}$.

Muchos niños presentan disfunciones cognitivas leves que no son detectadas y que posteriormente, al reingresar al sistema escolar, se hacen manifiestas. El trastorno cognitivo al no ser un déficit "evidente" tiende a subestimarse en esta población. Es fundamental que se realicen evaluaciones específicas de funciones ejecutivas, memoria y funcionamiento intelectual global, idealmente antes, durante y posterior a los tratamientos para documentar las alteraciones. Intervenciones de rehabilitación neurocognitiva, tanto no farmacológicas como farmacológicas, han demostrado mejorías en las evaluaciones cognitivas en estos pacientes ${ }^{16}$.

\section{Alteraciones endocrinas}

El grupo estudiado mostró que $26,6 \%$ de los niños presentaba algún trastorno hormonal diagnosticado en endocrinología. Pacientes con tumores de SNC fueron los más afectados $(40,0 \%)$. Casano ${ }^{21}$ describe que entre $40 \%$ $60 \%$ de los sobrevivientes llegan a presentar algún trastorno endocrino, siendo los más frecuentes, talla baja, compromiso gonadal e hipotiroidismo. En este mismo estudio, se observó que aquellos que recibieron radiación intracraneal presentaban aún más complicaciones endocrinas, principalmente debido a panhipopituitarismo secundario como secuela. La manifestación y el diagnóstico de trastornos endocrinos pueden ser tardíos y no estar presentes en etapas precoces, como probablemente sucedió en nuestro grupo.

\section{Trastornos sensoriales}

Se ha descrito que $13 \%$ de los niños con tumores de SNC y el 3\% de todos los tumores presentan compromiso visual severo ${ }^{14}$.

Estudios en población chilena han demostrado que pacientes que recibieron dosis elevadas de quimioterapia con cisplatino, radiación intracraneal o fueron tratados antes de los cinco años de edad, tienen mayor riesgo de desarrollar hipoacusia incluso después de 10 años de evolución ${ }^{22,23}$. Es importante destacar que uno de cada tres pacientes que ingresó al programa, presentaba algún tipo de trastorno visual y uno de cada cinco presentó hipoacusia. Los déficits sensoriales pueden dificultar la aplicación de evaluaciones (cognitivas, físicas y cuestionarios de fatiga) si éstas no están adaptadas para pacientes con visión o audición disminuida.

Tanto el trastorno visual como el trastorno cognitivo y auditivo son factores que pueden obstaculizar la rehabilitación. El equipo rehabilitador debe estar capacitado para reconocer la aparición de estos factores y poder trabajar con pacientes con estas condiciones.

\section{Fatiga relacionada al cáncer}

Tanto pacientes como padres han referido la fatiga como uno de los síntomas que causa más preocupación, y en este estudio corresponde a más del $40 \%$ de los ingresados. La presencia de fatiga puede obstaculizar las terapias de rehabilitación así como la adherencia a tratamientos. Estudios en distintos países han demostrado que la percepción de fatiga y calidad de vida se correlacionan. La prescripción de ejercicio, la promoción de higiene del sueño, la educación del paciente y su familia sobre como detectar y enfrentar la fatiga, son intervenciones que han demostrado disminuir la percepción de ésta ${ }^{24}$.

\section{Pruebas funcionales}

No existe un consenso sobre las pruebas funcionales más indicadas en este tipo de pacientes. Las utilizadas en este estudio evalúan la movilidad funcional (TUG test), el equilibrio (PBS) y el rendimiento cardiovascular (M6M). No hubo registro de resultados de estas pruebas en alrededor de la mitad de los pacientes, ya sea por edad o por limitaciones en la movilidad. Al sólo registrar valores de niños con menor discapacidad, se obtuvo un resultado "falsamente bueno" de las pruebas funcionales y no una imagen fidedigna de esta población. Este análisis nos hace reflexionar respecto a las escalas de evaluación funcional que empleamos en rehabilitación oncológica y en la necesidad de buscar alternativas a las pruebas estipuladas para los pacientes con mayor compromiso motor. 


\section{Conclusiones y desafíos futuros}

Podemos concluir que en la población de pacientes pediátricos chilenos que ingresan a rehabilitación oncológica encontramos dos perfiles predominantes. Por un lado, pacientes con tumores de SNC (generalmente de fosa posterior) con compromiso motor tipo síndrome cerebeloso más hemi/tetraparesia asociado a trastorno cognitivo. Con menor frecuencia, asociado a compromiso visual, auditivo y trastorno del habla.

Por otro lado, los pacientes con diagnóstico de leucemia y linfoma, tienen un perfil donde prima la presencia de fatiga y dolor. En muchos casos, éstas se deben a complicaciones asociadas, como polineuropatía por drogas o del paciente crítico y necrosis avascular de huesos largos ${ }^{25,26}$.

Se plantean como limitaciones del estudio la exclusión de pacientes por dificultades para contactarlos, la imposibilidad de realizarles evaluaciones ya sea por sus limitaciones motoras, visuales o por falta de cooperación. La ausencia de registro en estos casos puede alterar la estimación de la discapacidad en esta población. La realización de evaluaciones y exámenes confirmatorios es fundamental para objetivar y registrar la gran discapacidad que genera en estos pacientes y muchas veces no es posible realizarlo por falta de recursos.

Se requiere de más estudios para evaluar el impacto de estas deficiencias en los distintos dominios del funcionamiento en niños chilenos. El mejor conocimiento del paciente oncológico que ingresa a unidades especializadas de rehabilitación es clave para perfeccionar el modelo de funcionamiento de éstas ${ }^{27}$. Intervenciones para prevenir y disminuir la discapacidad en esta población, como el desarrollo de programas de vigilancia activa de deficiencias, impacto en la participación y programas de prehabilitación, son áreas que requieren desarrollo en este campo.

\section{Referencias bibliográficas}

1. Ferlay J, Soerjomataram I, Dikshit R, Eser S, Mathers $\mathrm{C}$, Rebelo $\mathrm{M}$, et al. Cancer incidence and mortal- ity worldwide: sources, methods and major patterns in GLOBOCAN 2012. Int J Cancer. 2015; 136 (5): E35986.

2. Ministerio de Salud, Chile. Primer Informe del Registro Nacional de Cáncer Infantil (RENCI) 2007-2011. 2018.

3. Darcy L, Enskär K, Granlund M, Simeonsson RJ, Peterson C, Björk M. Health and functioning in the everyday lives of young children with cancer: documenting with the International Classification of Functioning, Disability and Health-Children and Youth (ICF-CY) Child: Care, Health \& Development. 2015.

4. Pletschko, Felnhofer, Schwarzinger, Weiler, Slavc, Leiss. Applying the International Classification of Functioning-Children and Youth Version to Pediatric Neuro-oncology. J Child Neurol 2017; 32 (1): 23-8.

5. Wilson CL, Gawade PL, Ness KK. Impairments that influence physical function among survivors of childhood cancer. Child Basel Switz 2015; 2 (1): 1-36.

6. Gupta S, Rivera-Luna R, Ribeiro RC, Howard SC. Pediatric oncology as the next global child health priority: the need for national childhood cancer strategies in low- and middle-income countries. PLoS Med. 2014; 11 (6): e1001656.

7. Simioni C, Zauli G, Martelli AM, Vitale M, Ultimo $\mathrm{S}$, Milani D, et al. Physical training interventions for children and teenagers affected by acute lymphoblastic leukemia and related treatment impairments. Oncotarget. 2018; 9 (24): 17199-209.

8. Gamble GL, Gerber LH, Spill GR, Paul KL. The future of cancer rehabilitation: emerging subspecialty. Am J Phys Med Rehabil. 2011; 90 (5 Suppl 1): S76-87.

9. Ewertz M, Jensen AB. Late effects of breast cancer treatment and potentials for rehabilitation. Acta Oncol. 2011; 50 (2): 187-93.

10. Nicolini-Panisson RD, Donadio MVF. Normative values for the Timed "Up and Go" test in children and adolescents and validation for individuals with Down syndrome. Dev Med Child Neurol. 2014; 56 (5): 490-7.

11. Li AM, Yin J, Yu CCW, Tsang T, So HK, Wong E, et al. The six-minute walk test in healthy children: reliability and validity. Eur Respir J. 2005; 25 (6): 1057-60.

12. Franjoine MR, Gunther JS, Taylor MJ. Pediatric balance scale: a modified version of the berg balance scale for the school-age child with mild to moderate motor impairment. Pediatr Phys Ther Off Publ Sect Pediatr Am Phys Ther Assoc. 2003; 15 (2): 114-28.

13. Skinner R. Long-term effects of cancer therapy in children-functional effects, late mortality and longterm follow-up. Paediatr Child Health. 2012; 22 (6): 248-52. 
14. Silver JK, Baima J. Cancer prehabilitation: an opportunity to decrease treatment-related morbidity, increase cancer treatment options, and improve physical and psychological health outcomes. Am J Phys Med Rehabil. 2013; 92 (8): 715-27.

15. Brinksma A, Roodbol PF, Sulkers E, Kamps WA, de Bont ESJM, Boot AM, et al. Changes in nutritional status in childhood cancer patients: A prospective cohort study. Clin Nutr. 2015; 34 (1): 66-73.

16. Söntgerath R, Eckert K. Impairments of Lower Extremity Muscle Strength and Balance in Childhood Cancer Patients and Survivors: A Systematic Review. Pediatr Hematol Oncol. 2015; 32 (8): 585-612.

17. Castellino SM, Ullrich NJ, Whelen MJ, Lange BJ. Developing Interventions for Cancer-Related Cognitive Dysfunction in Childhood Cancer Survivors. JNCI J Natl Cancer Inst. 2014; 106 (8): dju186-dju186.

18. Mulhern RK, Butler RW. Neurocognitive sequelae of childhood cancers and their treatment. Pediatr Rehabil. 2004; 7 (1): 1-14; discussion 15-6.

19. Mulhern RK, Hancock J, Fairclough D, Kun L. Neuropsychological status of children treated for brain tumors: a critical review and integrative analysis. Med Pediatr Oncol. 1992; 20 (3): 181-91.

20. Geenen MM, Cardous-Ubbink MC, Kremer LCM, van den Bos C, van der Pal HJH, Heinen RC, et al. Medical assessment of adverse health outcomes in long-term survivors of childhood cancer. JAMA. 2007; 297 (24): 2705-15.

21. Dong Y, Crone C, Wise T. Chemobrain. Psychiatr Ann. 2014; 44 (7): 334-8.
22. Casano Sancho P. Endocrine sequelae in childhood cancer survivors. Endocrinol Diabetes Nutr. 2017; 64 (9): 498-505.

23. Waissbluth S, Chuang A, Del Valle Á, Cordova M. Long term platinum-induced ototoxicity in pediatric patients. Int J Pediatr Otorhinolaryngol. 2018; 107: 75-9.

24. Mujica-Mota M, Waissbluth S, Daniel SJ. Characteristics of radiation-induced sensorineural hearing loss in head and neck cancer: A systematic review: RadiationInduced Sensorineural Hearing Loss in Head and Neck Cancer. Head Neck. 2013; 35 (11): 1662-8.

25. Nunes MDR, Jacob E, Bomfim EO, Lopes-Junior LC, de Lima RAG, Floria-Santos M, et al. Fatigue and health related quality of life in children and adolescents with cancer. Eur J Oncol Nurs Off J Eur Oncol Nurs Soc. 2017; 29: 39-46.

26. Ness KK, Kaste SC, Zhu L, Pui C-H, Jeha S, Nathan $\mathrm{PC}$, et al. Skeletal, neuromuscular and fitness impairments among children with newly diagnosed acute lymphoblastic leukemia. Leuk Lymphoma. 2015; 56 (4): 1004-11.

27. Akyay A, Olcay L, Sezer N, Atay Sönmez Ç. Muscle strength, motor performance, cardiac and muscle biomarkers in detection of muscle side effects during and after acute lymphoblastic leukemia treatment in children. J Pediatr Hematol Oncol. 2014; 36 (8): 594-8.

28. Stout NL, Silver JK, Raj VS, Rowland J, Gerber L, Cheville A, et al. Toward a National Initiative in Cancer Rehabilitation: Recommendations From a Subject Matter. Expert Group. Arch Phys Med Rehabil 2016; 97 (11): 2006-15. 